\title{
BMJ Open Equity and intrapartum care by skilled birth attendant globally: protocol for a systematic review
}

\author{
Alisa Kachikis, ${ }^{1}$ Ann-Beth Moller, ${ }^{2}$ Tomas Allen, ${ }^{3}$ Lale Say, ${ }^{2}$ Doris Chou $^{2}$
}

To cite: Kachikis A, Moller A-B, Allen $\mathrm{T}$, et al. Equity and intrapartum care by skilled birth attendant globally: protocol for a systematic review. BMJ Open 2018;8:e019922. doi:10.1136/ bmjopen-2017-019922

- Prepublication history and additional material for this paper are available online. To view these files, please visit the journal online (http://dx.doi. org/10.1136/bmjopen-2017019922).

Received 3 October 2017 Revised 16 March 2018 Accepted 21 March 2018
Check for updates

${ }^{1}$ Department of Obstetrics and Gynecology, University of Washington, Seattle, Washington, USA

${ }^{2}$ Department of Reproductive Health and Research, World Health Organization, Geneva, Switzerland

${ }^{3}$ Library and Information Networks for Knowledge, World Health Organization, Geneva, Switzerland

Correspondence to

Dr Doris Chou; choud@who.int

\section{ABSTRACT}

Introduction Equity is a cross-cutting theme within the Sustainable Development Goals (SDGs) and central to the effort to improve maternal and child health globally. One key strategy to prevent maternal death set out in SDG 3 is assistance by a skilled birth attendant (SBA) at childbirth (indicator 3.1.2). However, the increased coverage of SBAs globally has not been reflected by the same degree of decrease in maternal mortality and has been reported to have higher levels of inequality than other maternal health interventions. There is a need to evaluate the extent of inequity in intrapartum care by SBAs and evaluate themes in determinants of inequity across regions and specific country characteristics.

Methods and analysis The protocol for this review follows The Cochrane Handbook for Systematic Reviews and Preferred Reporting Items for Systematic Reviews and Meta-Analyses with equity extension 2012 guidelines. Studies of all languages and from all countries from 2004, the year when the WHO/ICM/FIGO joint statement on SBAs was published, and onwards will be included. PubMed/ MEDLINE, CINAHL Complete, the Cochrane Library, POPLINE, the World Health Organization (WHO) Global Index Medicus, and grey literature will be searched. Our primary outcome is intrapartum care by SBA. Studies will be included if they evaluate equity and its determinants adapted from the Progress-Plus grouping of characteristics affecting health outcomes. Results will be stratified based on WHO, World Bank Group income and SDG regional groupings.

Ethics and dissemination This review is a secondary analysis of published literature and does not require ethics review. Results will provide information regarding equity in intrapartum care by SBAs globally and will inform development of indicators for monitoring of inequity as well as global policy related to intrapartum care and maternal mortality. Results will be disseminated via peer-reviewed manuscript, international conferences and stakeholder websites.

PROSPERO registration number CRD42017069021.

\section{INTRODUCTION}

Equity is a cross-cutting theme within the Sustainable Development Goals (SDGs) and central to the effort to move towards improved global maternal, child and adolescent health. ${ }^{2}$ Despite some progress during the Millennium Development Goals era,

\section{Strengths and limitations of this study}

This systematic review aims to evaluate equity in intrapartum care by skilled birth attendants (SBAs) globally, which will include specific determinants of inequity. No previous review of quantitative studies has evaluated equity/inequity in intrapartum care by SBAs during childbirth across the globe.

- The search strategy for this review is broad and comprehensive including studies in all languages and from all countries from multiple electronic databases, grey literature and websites.

- Intrapartum care by SBA as reported in observational studies may not report important nuances of care including comprehensiveness of care, time spent with the individual woman or quality of care.

- This literature review will evaluate a reproductive health indicator (intrapartum care by SBA) within the context of equity/inequity with attention to and expansion of validated tools to evaluate equity such as PROGRESS-Plus and the Preferred Reporting Items for Systematic Reviews and Meta- Analyses with equity extension framework.

- This review will summarise data collected using instruments not specifically designed to evaluate equity/inequity in relation to SBA intrapartum care; however, based on available data, it will help inform development of tools for future monitoring and evaluation of healthcare and equity related to SDG indicator 3.1.2.

preventable maternal mortality especially in low-income and middle-income countries (LMIC) has remained high. ${ }^{34}$ There is a need to focus on inequity and underlying social and structural determinants that contribute indirectly to maternal mortality. Special attention also needs to be paid to maternal mortality among high-risk groups such as adolescents and young women, particularly in humanitarian settings and in countries with armed conflict. $^{5}$

Factors associated with inequity across all countries include place of residence, gender/sex, socioeconomic status, education, as well as age. In specific regions or countries, migrant status, race, ethnicity, 
caste, religion can also be sources of inequity. ${ }^{2}$ SDG indicator 3.1 sets the target to reduce the global mortality ratio to less than 70 per 100000 live births by 2030 and one of the key strategies to prevent maternal deaths is assistance by a skilled birth attendant (SBA) at the time of childbirth, which is also reflected in the SDG indicator 3.1.2 'Births attended by skilled health personnel (\%)'. ${ }^{1} 67$ The SBA is defined in the joint statement by the WHO, the International Confederation of Midwives (ICM) and the International Federation of Gynaecology and Obstetrics (FIGO) as a 'midwife, doctor or nursewho has been educated and trained to proficiency in the skills needed to manage normal (uncomplicated) pregnancies, childbirth and the immediate postnatal period, and in the identification, management and referral of complications in women and newborns'. ${ }^{8}$ However, while the coverage of SBAs has increased globally, this has not been reflected in a proportional decrease in the global burden of maternal deaths. SBAs have been found to have a variable amount of knowledge and skills and, due to inconsistencies in data reporting, the definition of a SBA is currently under review. ${ }^{9-12}$ Nevertheless, around $50 \%$ of LMICs report that at least $80 \%$ rate of births are attended by SBAs. This number varies across countries. ${ }^{12}$ Inequity in SBA coverage has been found to be associated with economic status, education and place of residence and presence of SBA at birth has higher inequality rates than other maternal health interventions. ${ }^{12}$ This disparity is especially seen in LMICs where women in disadvantaged groups have SBA coverage rates of less than $50 \% .^{2}$

The definition of health inequity by Margaret Whitehead described disparities in health that are "not only unnecessary and avoidable but, in addition (...) unfair and unjust' and that have adverse effects on already disadvantaged groups within a population. ${ }^{13}{ }^{14}$ In addition, these health differences are systematic and not occasional or sporadic. ${ }^{14}$ In evaluating preventable maternal mortality and intrapartum care by SBAs globally as an indicator for maternal health, it is therefore important to assess the characteristics of women who are and who are not attended by an SBA at childbirth. This will allow for identification of possible determinants of inequity, development of potential interventions to address disadvantages and progress towards increased equity in maternal health.

\section{Study objectives and research questions}

The objectives of this study are to:

1. Conduct a systematic review of literature on intrapartum care by a SBA at childbirth and evaluate the extent of inequity that exists globally.

2. Identify determinants of inequity globally in intrapartum care by SBAs at the time of childbirth across regions and countries.

Our review seeks to answer the following research questions. (1) To what extent does inequality in intrapartum care by SBA exist globally? (2) What are determinants or themes of inequity that emerge globally and across countries and regions?

\section{METHODS AND ANALYSIS \\ Study registration}

This review protocol was registered with the Prospero database (registration number: CRD42017069021; date of registration: 26/06/2017).

\section{Study design}

The study method for this systematic review was developed based on the Preferred Reporting Items for Systematic Reviews and Meta-Analyses with equity extension (PRISMA-E 2012) guidelines, the PRISMA Protocols (PRISMA-P 2015) and The Cochrane Collaboration (Cochrane Handbook for Systematic Reviews). ${ }^{15-19}$ Please see online Supplementary file 1 for the PRISMA-E 2012 checklist and online Supplementary file 2 for the PRISMA-P 2015 Checklist. The literature search will follow the four-step flow diagram outline in the PRISMA statement. ${ }^{17}$

\section{Study eligibility criteria}

Inclusion criteria

Our research objectives will be assessed and studies selected based on criteria presented in table 1 .

\section{Population}

The population selected for this review include women of reproductive age who experienced childbirth within the timeframe of each individual research study. Surrogate search terms for this population centre on maternity care, pregnancy, childbirth, intrapartum care and obstetrics.

\begin{tabular}{|c|c|}
\hline Population & Women of reproductive age who experienced childbirth within the specified timeframe of an individual study. \\
\hline Intervention & Intrapartum care by skilled birth attendant (SBA) or institutional deliveries. \\
\hline Comparison & Utilisation of non-SBA or traditional birth attendants at the time of childbirth as well as unattended births. \\
\hline Outcome & $\begin{array}{l}\text { Evaluation of inequity in provision of intrapartum care by SBA at the time of childbirth with quantitative } \\
\text { evaluation of determinants of inequity. }\end{array}$ \\
\hline Study design & $\begin{array}{l}\text { All observational quantitative studies (including but not limited to prospective and retrospective cohort studies, } \\
\text { cross-sectional studies, mix-methods studies). }\end{array}$ \\
\hline Context & $\begin{array}{l}\text { All countries with health facility and/or community-based services offering childbirth care. Years of publication } \\
\text { ranging from } 1 \text { January } 2004 \text { to the time of this study. }\end{array}$ \\
\hline
\end{tabular}




\section{Intervention/comparison}

Studies reporting intrapartum care by SBA with or without comparison to utilisation of non-skilled or traditional birth attendants as well as unattended births will be included in this review. Intrapartum care is defined as care during the labour and childbirth of a woman which includes immediate post-delivery care around the third stage of labour. Intrapartum care by an SBA may be indicated with the presence of SBA at the time of childbirth or by access to SBA whether or not a woman chooses to utilise care by an SBA. Institutional delivery may be used as a proxy for SBA since the concept of SBA and their skill level, competency, education and accreditation are currently inconsistent across countries. ${ }^{9} 11$ For the purposes of this study, SBA is defined as a skilled health professional who is qualified by education and training and has skills proficient to provide intrapartum and immediate postpartum interventions with the goal of improving maternal and newborn health. ${ }^{8}$ The purpose of this study, however, is not to evaluate the qualifications, competency or skills of specific SBA cadres in each study.

\section{Outcome}

We will include studies evaluating inequity in intrapartum care by an SBA. Alternative search terms include but are not limited to disparities, inequality and barriers to care. Given the moral imperative of the word equity, additional search terms such as social justice will also be utilised. ${ }^{20}$ Based on prior reviews of inequity in maternal care, determinants of inequity are hypothesised to include demographic factors such age race/ethnicity, socioeconomic status, place of residence or geographic factors, as well as reproductive indicators. ${ }^{12} 21-23$ For the purposes of this review, studies will be included if they compare at least two populations by one or more indicators. PROGRESS-Plus, an established framework for sociodemographic factors that may contribute to inequity in health, is an acronym that stands for place of residence, race/ethnicity/culture and language, occupation, gender/sex, religion, education, socioeconomic status, social capital while 'Plus' adds three additional context-specific factors including personal characteristics that attract discrimination, features of relationships and time-dependent relationships. ${ }^{24-26}$ The PROGRESS-Plus framework has been adapted for the purposes of this project to include indicators specific to gender, sexual and reproductive health, including key indicators of the Global strategy for Women's, Children's and Adolescents' Health (2016-2030) and Strategies towards Ending Preventable Maternal Mortality core maternal health indicators. ${ }^{27-34}$

\section{Study design}

All observational quantitative or semiquantitative studies of any design will be included if they evaluate inequity in intrapartum care by SBAs which includes barriers to care, disparities or similar.

\section{Context}

Studies of all languages and across all settings with health facility and/or community-based services offering childbirth care will be eligible for inclusion. Studies published from 2004 until the time of this review will be considered given the increased global interest in maternal health and SBAs after the United Nations' Millennium Declaration in 2000 and establishment of the Millennium Development Goals and WHO, ICM and FIGO's joint statement on the importance of SBAs in $2004 .^{835}$

\section{Exclusion criteria}

Studies will not be eligible for inclusion if:

1. There are no details regarding determinants that may indicate inequity (e.g., demographic factors, socioeconomic factors, reproductive history, geography and so on).

2. Only qualitative data is collected.

3. They are systematic reviews.

4. The sample selection and size does not provide results generalisable to the general population or a significant subgroup of the population (e.g., a country's second-level administrative division).

\section{Search strategy}

In order to answer our research questions, a search for all literature based on related search terms will be conducted using the following online bibliographic databases: PubMed/MEDLINE, CINAHL Complete, POPLINE, the Cochrane Database of Systematic Reviews and the WHO Global Index Medicus (GIM). In addition, a manual search of bibliographic references of retrieved studies and systematic reviews as well as grey literature of international organisations and websites relevant to the field of maternal and child health will be conducted, including, but not limited, to National Institute for Health and Clinical Excellence, National Institute of Health, United Nations Children's Fund, United Nations Population Fund and WHO. The search will be inclusive of all languages and will be conducted with specific search terms related to (1) childbirth; (2) SBA, non-SBA, facility and non-facility deliveries; (3) equity and (4) utilisation of care or access. Please see online Supplementary file 3 for the detailed search strategy for PubMed/Medline and the Cochrane Library.

\section{Study selection}

Following a comprehensive and detailed literature search, all duplicate articles will be removed. A team of two $(\mathrm{AK} / \mathrm{ABM})$ will screen titles and abstracts of retrieved studies for relevance and eligibility for inclusion. Disagreements will be resolved by an additional reviewer (DC). All study abstracts selected for inclusion will then undergo an independent full-text review with similar methodology. All chosen full-text articles will then be evaluated again for inclusion based on inclusion and exclusion criteria by all co-authors and studies that do not meet the criteria will be eliminated from the 
study. Disagreements will be resolved by mutual agreement. Full-text articles in languages other than English will be translated.

\section{Data extraction}

A standardised data collection form was used as a template for development of a data extraction form for this review. ${ }^{36}$ Study details collected include but are not limited to study characteristics (country, year and journal of publication, study design and dates), population and setting (population description, setting description, inclusion/exclusion criteria, sample size), intervention (SBA and non-SBA cadres, facility description), equity measures (primary outcome, determinants, results, study quality and conclusions). During the review, additional fields on the data extraction form will allow for flexibility for additional data points or determinants as needed. Two reviewers will independently extract data from the selected studies and discrepancies will be discussed with a third reviewer. Please see online Supplementary file 4 for the data extraction form.

\section{Scientific quality assessment}

Scientific quality of studies will be assessed based on the Effective Public Health Practice Project's quality assessment tool for quantitative studies which includes an assessment for study bias. ${ }^{37}$ Study methodology will be classified as strong, moderate, or weak. Two reviewers (AK/ABM) will independently evaluate each study and will resolve conflicts by reviewing the articles together. Degree of bias will be reported in the results.

\section{Analysis plan}

We will evaluate quantitative measures of relations between possible determinants of inequity and intrapartum care by SBA or institutional delivery. Results reported in published studies may include proportions, means, percentages, rates or other quantifiable differences between two or more groups. Methodology for analysing health disparities will be followed according to published guidelines. ${ }^{38} 39$ All research studies reporting secondary analyses of nationally representative household surveys will be reviewed and only studies reporting the most recent survey from an individual country will be considered. Subanalyses may include evaluation of inequity based on different measures of intrapartum care by SBA intrapartum care or institutional deliveries. Results will be stratified based on WHO, World Bank Group income and SDG regional groupings. ${ }^{40-42}$ Data will be presented in tables by study, country, region and/or theme.

\section{Patient and public involvement}

Patients and the public were not involved in the development of this protocol. This systematic review of published literature will not involve recruitment and participation of patients.

\section{DISSEMINATION AND ETHICS}

This systematic review will provide information regarding equity and determinants of inequity in intrapartum care by SBAs globally. It will inform the development of indicators for monitoring and evaluation of inequity in intrapartum care by SBAs globally, which is pertinent given the higher level of inequality reported with this maternal health intervention. ${ }^{12}$ This work is also especially relevant given the current effort to revise the definition for SBA and measuring tools by collaborating international and professional organisations. This work will also guide establishment of global policy on health equity specifically related to intrapartum care by SBA and maternal mortality. The provision and presence of SBAs and utilisation and access to their services is essential in decreasing maternal and newborn mortality globally.

Final study results will be disseminated via a peer-reviewed publication, which will include all supplemental materials on search strategy, data extraction, compilation and analysis. This systematic review is a secondary analysis of previously published literature and therefore does not require ethics review and approval.

Contributors DC is the guarantor of this review. AK, ABM, DC and LS contributed to the initial conception and design of this systematic review. AK, ABM, TA and DC developed the search strategies. AK drafted the proposal manuscript. All authors participated in critically revising the protocol for intellectual content. All authors read and approved the final manuscript.

Funding This project was supported by a Queenan Fellowship for Global Health grant from the Foundation for SMFM, the non-profit arm of the Society for MaternalFetal Medicine (SMFM). This work was also funded by the UNDP-UNFPA-UNICEFWHO-World Bank Special Programme of Research, Development and Research Training in Human Reproduction (HRP), a co-sponsored program executed by the WHO and this publication was supported by a subgrant from JHSPH with funds provided by Grant No. OPP1096225 from The Bill and Melinda Gates Foundation.

Disclaimer The authors alone are responsible for the views expressed in this article and they do not necessarily represent the views, decisions or policies of the funding bodies or institutions with which they are affiliated.

Competing interests None declared.

Patient consent Not required.

Provenance and peer review Not commissioned; externally peer reviewed.

Data sharing statement All data generated or analysed during this study will be included in the published article and its supplementary information files.

Open Access This is an open access article distributed under the terms of the Creative Commons Attribution-NonCommercial IGO License (CC BY-NC 3.0 IGO), which permits use, distribution, and reproduction for non-commercial purposes in any medium, provided the original work is properly cited. In any reproduction of this article there should not be any suggestion that WHO or this article endorse any specific organization or products. The use of the WHO logo is not permitted. This notice should be preserved along with the article's original URL. See:https:// creativecommons.org/licenses/by-nc/3.0/igo

(C) Article author(s) (or their employer(s) unless otherwise stated in the text of the article) 2018. All rights reserved. No commercial use is permitted unless otherwise expressly granted.

\section{REFERENCES}

1. Sustainable Development Knowledge Platform. United Nations. 2017 https://sustainabledevelopment.un.org/ (accessed 14 June 2017).

2. World Health Organization. World health statistics 2017: monitoring health for the SDGs, Sustainable Development Goals. Geneva: World Health Organization, 2017. 
3. Say L, Chou D, Gemmill A, et al. Global causes of maternal death: a WHO systematic analysis. Lancet Glob Health 2014;2:e323-33.

4. Alkema L, Chou D, Hogan D, et al. Global, regional, and national levels and trends in maternal mortality between 1990 and 2015 with scenario-based projections to 2030: a systematic analysis by the UN Maternal Mortality Estimation Inter-Agency Group. Lancet 2016;387:462-74.

5. Gaffey MF, Das JK, Bhutta ZA. Millennium development goals 4 and 5: past and future progress. Semin Fetal Neonatal Med 2015;20:285-92.

6. United Nations Statistics Division. Metadata for the proposed global indicators for the review of the 2030 agenda for sustainable development: metadata for goal 3: United Nations. 2016 http:// unstats.un.org/sdgs/files/metadata-compilation/Metadata-Goal-3.pdf (cited 11 March 2017)

7. Every Woman Every Child. Global strategy for women's, children's and adolescents' health (2016-2030). New York, 2015.

8. World Health Organization (WHO). International Confederation of Midwives (ICM), International Federation of Gynaecology and Obstetrics (FIGO). Making pregnancy safer: the critical role of the skilled attendant - a joint statement by WHO, ICM and FIGO. Geneva: WHO, 2004.

9. World Health Organization (WHO). Draft statement on skilled attendant at birth: WHO. 2017 http://www.who.int/ reproductivehealth/skilled-birth-attendant/en/ (accessed 11 March 2017).

10. Harvey SA, Ayabaca P, Bucagu M, et al. Skilled birth attendant competence: an initial assessment in four countries, and implications for the Safe Motherhood movement. Int J Gynaecol Obstet 2004;87:203-10.

11. Harvey SA, Blandón YC, McCaw-Binns A, et al. Are skilled birth attendants really skilled? A measurement method, some disturbing results and a potential way forward. Bull World Health Organ 2007:85:783-90.

12. World Health Organization (WHO). State of inequality: reproductive, maternal, newborn and child health. Geneva: WHO, 2015.

13. Whitehead M. The concepts and principles of equity and health. Int $J$ Health Serv 1992;22:429-45.

14. Braveman P. Health disparities and health equity: concepts and measurement. Annu Rev Public Health 2006;27:167-94.

15. Welch V, Petticrew M, Tugwell P, et al. PRISMA-equity 2012 extension: reporting guidelines for systematic reviews with a focus on health equity. PLoS Med 2012;9:e1001333.

16. Welch V, Petticrew M, Petkovic J, et al. Extending the PRISMA statement to equity-focused systematic reviews (PRISMA-E 2012): explanation and elaboration. Int J Equity Health 2015;14:92.

17. Moher D, Liberati A, Tetzlaff J, et al. Preferred reporting items for systematic reviews and meta-analyses: the PRISMA statement. $J$ Clin Epidemiol 2009;62:1006-12.

18. The Cochrane Collaboration. Cochrane handbook for systematic reviews of interventions version 5.1.0, 2011.

19. Moher D, Shamseer L, Clarke M, et al. Preferred reporting items for systematic review and meta-analysis protocols (PRISMA-P) 2015 statement. Syst Rev 2015;4 1:1.

20. Braveman P, Gruskin S. Defining equity in health. J Epidemiol Community Health 2003;57:254-8.

21. Langlois ÉV, Miszkurka M, Zunzunegui MV, et al. Inequities in postnatal care in low- and middle-income countries: a systematic review and meta-analysis. Bull World Health Organ 2015;93:259-70.

22. Çalıșkan Z, Kılıç D, Öztürk S, et al. Equity in maternal health care service utilization: a systematic review for developing countries. Int $J$ Public Health 2015;60:815-25.
23. Say L, Raine R. A systematic review of inequalities in the use of maternal health care in developing countries: examining the scale of the problem and the importance of context. Bull World Health Organ 2007;85:812-9.

24. O'Neill J, Tabish $\mathrm{H}$, Welch $\mathrm{V}$, et al. Applying an equity lens to interventions: using PROGRESS ensures consideration of socially stratifying factors to illuminate inequities in health. $J$ Clin Epidemio 2014;67:56-64.

25. Evans T, Brown H. Road traffic crashes: operationalizing equity in the context of health sector reform. Inj Control Saf Promot 2003;10:11-12

26. Oliver S, Dickson K, Newman M. Getting started with a review. In: Gough D, Oliver S, Thomas J, eds. An introduction to systematic reviews. London, UK: SAGE Publications, 2012.

27. Moran AC, Jolivet RR, Chou D, et al. A common monitoring framework for ending preventable maternal mortality, 20152030: phase I of a multi-step process. BMC Pregnancy Childbirth 2016;16:250.

28. Every Woman Every Child. Indicator and monitoring framework for the Global Strategy for Women's, Children's and Adolescents' Health (2016-2030), 2016.

29. MEASURE Evaluation. Compendium of gender equality and HIV indicators. Compendium of gender equality and HIV indicators. 2014 http://www.cpc.unc.edu/measure/publications/ms-13-82 (cited 2017 June 13).

30. United Nations Statistics Division. The United Nations minimum set of gender indicators. 2017 https://genderstats.un.org/files/ Minimum\%20Set\%20indicators\%20web.pdf (cited 2017 June 13).

31. The DHS Program. Women's status and empowerment. http://www. dhsprogram.com/topics/Womens-Status-and-Empowerment.cfm (accessed 13 June 2017).

32. World Health Organization. Measuring sexual health: conceptual and practical considerations and related indicators, 2010.

33. World Health Organization. National-level monitoring of the achievement of universal access to reproductive health: conceptual and practical considerations and related indicators. Geneva WHO, 2008.

34. UNAIDS. Global aids response progress reporting 2014: construction of core indicators for monitoring the 2011 United Nations Political Declaration on HIV and AIDS, 2014.

35. United Nations. United Nations millennium declaration. New York, USA: United Nations, 2000

36. Effective Practice and Organisation of Care (EPOC). EPOC resources for review authors. Oslo: Norwegian Knowledge Centre for the Health Services, 2015.

37. Effective Public Health Practice Project. Quality assessment tool for quantitative studies. Hamilton, ON: Effective Public Health Practice Project, 1998.

38. Keppel KG, Pearcy JN. Measuring relative disparities in terms of adverse events. J Public Health Manag Pract 2005;11:479-83.

39. Tugwell P, Petticrew M, Kristjansson E, et al. Assessing equity in systematic reviews: realising the recommendations of the commission on social determinants of health. BMJ 2010;341:c4739.

40. WHO. WHO regional offices: world health organization. $2017 \mathrm{http}: / /$ www.who.int/about/regions/en/ (accessed 22 June 2017).

41. The World Bank Group. World bank country and lending groups. https://datahelpdesk.worldbank.org/knowledgebase/articles/906519world-bank-country-and-lending-groups (accessed 22 June 2017).

42. United Nations Statistics Division. SDG indicators: regional groupings used in 2017 report and statistical annex. $2017 \mathrm{https}: / /$ unstats.un.org/sdgs/indicators/regional-groups/ (accessed 22 June 2017). 